\title{
Thermodynamics of Dark Energy
}

\author{
Neven Bilić \\ Rudjer Bošković Institute, 10002 Zagreb, Croatia \\ E-mail: bilic@thphys.irb.hr
}

August 27, 2018

\begin{abstract}
Thermodynamic properties of dark energy are discussed assuming that dark energy is described in terms of a selfinteracting complex scalar. We first show that, under certain assumptions, selfinteracting complex scalar field theories are equivalent to purely kinetic $k$-essence models. Then we analyze the themal properties of $k$-essence and in particular we show that dark-energy in the phantom regime does not necessarily yield negative entropy.
\end{abstract}

\section{Introduction}

A motivation for and importance of studying the dark energy (DE) thermodynamics can be summarized in the following questions: Does a DE fluid possess thermal, besides hydrodynamical, properties such as temperature? What is the thermodynamical fate of the Universe? Can DE cluster? In a number of recent papers [1, 2, 3, 4, 15, 6, 7, 8, 9, thermal properties of dark energy have been discussed based on the assumption that the dark energy substance is a thermalized ensemble at certain temperature with an associate thermodynamical entropy. Here we hope to shed some light on a rather peculiar thermodynamic related properties of DE often discussed in the literature. These may be illustrated by a few statements taken from recent papers: DE becomes hotter if it undergoes an adiabatic expansion [2]. Phantom DE violates the null energy condition and hence either its entropy or its temperature must be negative [5, 8]. A negative temperature implies either that the phantom should be quantized or that its space-time should be Euclidean [3].

The essential feature of DE is that its pressure must be negative in order to reproduce the accelerated expansion of the Universe. Hence, the DE equation of state violates the strong energy condition. The simplest DE model, the cosmological constant, is basically the vacuum energy, with the equation of state $p=-\rho$. As a consequence, in the course of the Universe evolution, the vacuum energy density remains constant and its thermal properties are trivial. In other DE models with more complicated equation of state (EOS) the energy density varies with time. A number of models, such as quintessence [10] and k-essence [11], are based on scalar field theories. To this class belong also the so called quartessence models [19], where the term quartessence was invented to denote the unification of dark energy and 
dark matter. A very popular class of models is the phantom DE, the EOS of which violates even the null energy condition (NEC), i.e, in these models $(p+\rho)<0$. For an excellent recent review of DE models, see [12]).

Dark energy is usually described by an EOS in the barotropic form, i.e., in the form $p=p(\rho)$. Equivalently, one may define a field theory Lagrangian, in which dark energy is described in terms of a classical selfinteracting field coupled to gravity. Then, the EOS may be deduced from the energy-momentum tensor obtained from variational principle. From the EOS alone it is not possible to uniquely determine the thermodynamic properties of a system. One simple example is the $\operatorname{EOS} p=\rho / 3$ which may describe a massless boson gas at $T \neq 0$ (hence $S \neq 0$ ) but also a massless degenerate Fermi gas at $T=0$ (hence $S=0$ ). A similar situation occurs for any barotropic EOS.

The purpose of this paper is twofold. First, we would like to demonstrate that, under reasonable assumption in the cosmological context, the ghost condensate and purely kinetic $k$-essence models are equivalent to standard, selfinteracting complex scalar field theories. Second, we analyze the thermal properties of a grand canonical DE gas described by purely kinetic k-essence with a chemical potential that corresponds to a conserved charge. The conserved charge $Q$ is related to the shift symmetry $\phi \rightarrow \phi+$ const which corresponds to to the $U(1)$ symmetry of the equivalent complex scalar field theory. Hence, the conserved quantity $Q$ corresponds to the usual $U(1)$ charge of a complex scalar field. In this way a consistent grandcanonical description of DE involves the thermodynamic equations with two variables: the temperature $T$ and chemical potential $\mu$.

\section{Thomas-Fermi correspondence}

Consider the Lagrangian

$$
\mathcal{L}=\eta g^{\mu \nu} \Phi_{, \mu}^{*} \Phi_{, \nu}-V\left(|\Phi|^{2} / m^{2}\right)
$$

for a complex scalar field

$$
\Phi=\frac{\phi}{\sqrt{2}} \exp (-i m \theta)
$$

where the potential $V$ may contain the mass term $m^{2}|\Phi|^{2}$ and higher order selfinteraction terms. Here $\eta=1$ for a canonical scalar field and $\eta=-1$ for a phantom. The field $\Phi$ satisfies the Klein-Gordon equation of motion

$$
\frac{1}{\sqrt{-g}}\left(\sqrt{-g} g^{\mu \nu} \Phi_{, \nu}\right)_{, \mu}+\frac{d V}{d|\Phi|^{2}} \Phi=0
$$

If the space-time variations of $|\Phi|$ are small on the scale smaller than $m^{-1}$, i.e. assuming $\phi_{, \mu} \ll m \phi$, then the Thomas-Fermi (TF) approximation [13, 15] applies and the derivatives of $\Phi$ may be calculated as $\Phi_{, \mu}=-i m \Phi \theta_{, \mu}$. Hence, neglecting $g^{\mu \nu} \phi_{, \mu} \phi_{, \nu} / m^{2}$, the Lagrangian (1) may be written as

$$
\mathcal{L}_{\mathrm{TF}} / m^{4}=X Y-U(Y),
$$

where we have introduced the abbreviations

$$
X=g^{\mu \nu} \theta_{, \mu} \theta_{, \nu} ; \quad Y=\eta \frac{\phi^{2}}{2 m^{2}},
$$


and the dimensionless potential

$$
U(Y)=\frac{1}{m^{4}} V(\eta Y) .
$$

The equations of motion for the field $\phi$ and $\theta$ are

$$
\begin{gathered}
X-U_{Y}=0, \\
\left(Y g^{\mu \nu} \theta_{, \nu}\right)_{; \mu}=0,
\end{gathered}
$$

where $U_{Y}=d U / d Y$. The set of equations (7), (8) is basically a reduced Klein-Gordon equation (3). Equation (5) implies $\eta Y>0$. Assuming $X>0$ and hence

$$
U_{Y}>0,
$$

the field $\theta$ may be treated as a velocity potential for the fluid 4-velocity

$$
u^{\mu}=g^{\mu \nu} \theta_{, \nu} / \sqrt{X},
$$

satisfying the normalization condition $u_{\mu} u^{\mu}=1$. As a consequence, the energy-momentum tensor $T^{\mu \nu}$ obtained from the Lagrangian (4)

$$
T_{\mu \nu}=2 \frac{\partial \mathcal{L}_{\mathrm{TF}}}{\partial X} \theta_{, \mu} \theta_{, \nu}-\mathcal{L}_{\mathrm{TF}} g_{\mu \nu}
$$

takes the perfect fluid form,

$$
T_{\mu \nu}=(\rho+p) u_{\mu} u_{\nu}-p g_{\mu \nu}
$$

with the parametric equation of state

$$
\rho / m^{4}=Y U_{Y}+U, \quad p / m^{4}=Y U_{Y}-U .
$$

A perfect fluid description applies only if (9) holds which, generally, may not be true for the entire range $0 \leq \eta Y \leq \infty$. For a canonical field $(\eta=1)$ the fluid is perfect for those $Y$ for which $d V / d|\Phi|^{2}>0$. In contrast, a phantom field $(\eta=-1)$ behaves as a perfect fluid when $d V / d|\Phi|^{2}<0$.

Owing to (7) and the obvious relation $\partial \mathcal{L}_{\mathrm{TF}} / \partial X=m^{4} Y$ equation (4) may be written as a Legendre transformation

$$
W(X)+U(Y)=X Y
$$

with

$$
\begin{gathered}
X=U_{Y} \\
Y=W_{X}
\end{gathered}
$$

where $W_{X}=d W / d X$. Equation (14) with (15) and (16) defines an equivalence relation. We say that potentials $W(X)$ and $U(Y)$ are $T F$ equivalent to each other. Given $U \equiv V(\eta Y) / m^{4}$, the potential $W(X)$ can be found by solving (15) for $Y$ and plugging the solution in (14). More explicitly

$$
W(X)=X U_{Y}^{-1}(X)-U\left(U_{Y}^{-1}(X)\right),
$$

where $U_{Y}^{-1}$ is the inverse function of $U_{Y}$. Similarly, if $W(X)$ is known, the potential $U$ may be derived in the same way. 
The potential $W(X)$ that is TF equivalent to $U(Y)$ describes a field theory with the Lagrangian

$$
\mathcal{L}=m^{4} W(X) ; \quad X \equiv g^{\mu \nu} \theta_{, \mu} \theta_{, \nu}
$$

which depends only on the derivatives of a scalar field $\theta$. The equation of motion for $\theta$

$$
\left(W_{X} g^{\mu \nu} \theta_{, \nu}\right)_{; \mu}=0
$$

is equivalent to (8). However, the field theories described by the Lagrangians (4) and (18), respectively, are equivalent only at the classical level, since the Lagrangian (18) is obtained from (44) by eliminating one degree of freedom with help of the equation of motion (77). Quantum mechanically, these two field theories describe different physics.

The energy-momentum tensor constructed from the Lagrangian (18) is of the form (12), with the parametric equation of state

$$
\rho / m^{4}=2 X W_{X}-W, \quad p / m^{4}=W .
$$

This equation describes an ordinary fluid for $W_{X}>0$ and phantom for $W_{X}<0$. The above mentioned equivalence between the two theories may be seen by noting that equations (13) and (20) are different parameterization of the same equation of state. This may be easily verified by applying (15) as a reparameterization of (20).

Consider a few examples:

\subsection{Higgs potential}

The quartic potential for a complex scalar field is given by

$$
V_{4}(\Phi)=V_{0} \pm m^{2}|\Phi|^{2}+\lambda|\Phi|^{4}
$$

where the - sign in front of the mass term gives the Higgs potential. The potential may be written as $V_{4}=V_{0}+V_{ \pm}\left(|\Phi|^{2} / m^{2}\right)$ with

$$
U_{ \pm}(Y) \equiv \frac{1}{m^{4}} V_{ \pm}(\eta Y)=\lambda\left(\eta Y \pm \frac{1}{2 \lambda}\right)^{2}-\frac{1}{4 \lambda} .
$$

Solving (14) we find

$$
W_{ \pm}(X)=\frac{1}{4 \lambda}(\eta X \mp 1)^{2} .
$$

In the example of ghost condensate explored in [14] the potential $W(X)=(X-1)^{2}$ defined on the domain $X>1$ is TF equivalent to the canonical quartic potential $V_{+}$with $\eta=1$. The same potential on the $X<1$ domain is TF equivalent to the phantom Higgs potential $V_{-}$with $\eta=-1$.

\subsection{Chaplygin gas}

In contrast to the standard assumption that dark matter and dark energy are distinct, there stands the hypothesis that both are different manifestations of a single entity. The first 
definite model of this type [16, 15, 17] is based on the Chaplygin gas, an exotic fluid with an equation of state

$$
p=-\frac{A}{\rho}
$$

Subsequently, the generalization to

$$
p=-\frac{A}{\rho^{\alpha}} ; \quad 0 \leq \alpha \leq 1
$$

was given [18] and the term 'quartessence' coined [19] to describe unified dark matter/energy models.

One of the most appealing aspects of the original Chaplygin gas model is that it is equivalent to the Dirac-Born-Infeld description of a D-brane in string theory. [20, 21]. This may be seen as follows [22]. Consider a $p$-dimensional D-brane with coordinates $x^{\mu}, \mu=$ $0,1 \ldots p$, moving in the $\mathrm{p}+1$-dimensional bulk with coordinates $X^{a}, a=0,1 \ldots p+1$. In the string frame the action is given by [23]

$$
S_{\mathrm{DBI}}=-\sqrt{A} \int d^{p+1} x \sqrt{(-1)^{p} \operatorname{det} g^{(\mathrm{ind})}}
$$

where $g_{\mu \nu}^{(\text {ind })}$ is the induced metric or the "pull back" of the bulk space-time metric $G_{a b}$ to the brane,

$$
g_{\mu \nu}^{(\mathrm{ind})}=G_{a b} \frac{\partial X^{a}}{\partial x^{\mu}} \frac{\partial X^{b}}{\partial x^{\nu}} .
$$

Let us choose the coordinates such that $X^{\mu}=x^{\mu}$ and let the $p+1$-th coordinate $X^{p+1} \equiv \theta$ be normal to the brane. From now on we set $p=3$ and consider a 3 -brane universe in a $4+1$ dimensional bulk. Then

$$
G_{\mu \nu}=g_{\mu \nu} ; \quad \mu=0, \ldots, 3 ; \quad G_{\mu 4}=0 ; \quad G_{44}=-1
$$

After a few straightforward algebraic manipulations, the DBI action may be written as

$$
S_{\mathrm{DBI}}=\int d^{4} x \sqrt{-\operatorname{det} g} \mathcal{L}_{\mathrm{DBI}} ; \quad \mathcal{L}_{\mathrm{DBI}}=-\sqrt{A} \sqrt{1-X}
$$

where $X$ is given by (5). The energy-momentum tensor constructed from this Lagrangian takes the perfect fluid form (12) with

$$
\rho=\frac{\sqrt{A}}{\sqrt{1-X}} \quad p=-\sqrt{A} \sqrt{1-X}
$$

From this the Chaplygin gas equation. of state (24) follows.

Using $W(X)=\mathcal{L}_{\mathrm{DBI}} / m^{4}$, where $m^{4}=\sqrt{A} / 2$, we find the TF equivalent

$$
U(Y)=Y+\frac{1}{Y}
$$




\subsection{Symmetry breaking and condensation}

Consider the Lagrangian (11) for a complex scalar field with the potential $V(y) \geq 0$ that possesses a minimum at some point $y_{0} \equiv \phi_{0}^{2} /\left(2 m^{2}\right)$, i.e., assume that there exists a point where $d V / d y=0$ and $d^{2} V / d y^{2}>0$. In this case a spontaneous breakdown of $\mathrm{U}(1)$ symmetry of the Lagrangian (11) will take place. One of the components of the field $|\Phi|$ will have a nonzero vacuum expectation value such that the classical part of the field satisfies $|\Phi|^{2}=$ $\phi_{0}^{2} / 2$. There will be two quantum modes fluctuating around the minimum, one of them massive and the other one massless (Goldston boson). A typical example is the Higgs potential $V_{4}$ in (21) with the "wrong" sign of the mass term. The minima are obviously placed at positions satisfying $\phi_{0}^{2}=m_{0}^{2} / \lambda$. Another example is the Chaplygin gas potential (31)

$$
V_{\mathrm{Ch}}=m^{4}\left(\frac{|\Phi|^{2}}{m^{2}}+\frac{m^{2}}{|\Phi|^{2}}\right)
$$

with the minima at $\Phi_{0}^{2}=m^{2}$. In the neighborhoods of the minima, this potential closely resembles (21) which may be seen by expanding (32) around a minimum and identifying $V_{0} \equiv 3 m^{4}, m_{0}^{2} \equiv 2 m^{2}$, and $\lambda \equiv 1$.

In the neighborhood of a minimum, i.e., of a point $y_{0}$ where $d V / d y=0$ and $d^{2} V / d y^{2}>0$, the solution will adiabatically role towards the minimum. Hence, we may assume that the solution is almost static. One finds three phases of the condensate corresponding to three types of solutions according to the sign of $d V / d y$ :

i) $y>y_{0}$. In this region $d V / d y>0$. A configuration that solves Eqs. (77) and (8) may be represented in terms of a self gravitating perfect fluid with the 4-velocity

$$
u^{\mu}=g^{\mu \nu} \theta_{, \nu}\left(\frac{1}{m^{4}} \frac{d V}{d y}\right)^{-1 / 2}
$$

and with the equation of state given in a parametric form (13). In this case the dominant energy condition $\rho \geq 0 ; \quad \rho \pm p \geq 0$ holds and the corresponding set of solutions describe a canonical phase.

The reduced Klein-Gordon equation (7), (8) may be further simplified in the comoving reference frame, i.e., in the frame where the 4 -velocity takes the form $u^{\mu}=\delta_{0}^{\mu} / \sqrt{g_{00}}$. As a consequence of this and (33) the field $\theta$ is now a function of $t$ only. Furthermore, Eq. (8) gives

$$
\theta=\frac{\mu}{m} t+\text { const }
$$

where the constant $\mu$ is the chemical potential associated to the conservation of $\mathrm{U}(1)$ charge [24, 25]. Equation (7) degenerates now into an algebraic equation

$$
\mu^{2} g^{00}-\frac{1}{m^{2}} \frac{d V}{d y}=0
$$

which relates the metric to the field $\phi$. The quantity $\mu$ is introduced in the Euclidean path integral by replacing the derivative with respect to the Euclidean time $\tau=i t$ with

$$
\frac{\partial}{\partial \tau} \rightarrow \frac{\partial}{\partial \tau} \pm \mu
$$


where the + or $-\operatorname{sign}$ is taken when the derivative acts on $\Phi^{*}$ or $\Phi$, respectively.

ii) $y<y_{0}$. In this region $d V / d y<0$. The procedure similar to i) may be repeated for $\eta=-1$. The velocity of the fluid is now defined as

$$
u^{\mu}=g^{\mu \nu} \theta_{, \nu}\left(-\frac{1}{m^{4}} \frac{d V}{d y}\right)^{-1 / 2},
$$

and we have a perfect fluid with the EOS given by (13) as before but $\rho+p<0$ so that the null and the dominant energy conditions are now violated. In the neighborhood of the minimum the field $\theta$ is again given by (34) and the chemical potential is defined by

$$
m^{2} \mu^{2} g^{00}+\frac{d V}{d y}=0
$$

The solutions to (7), (8) represent the Bose-Einstein condensate of the phantom field. Hence, this type of solutions may be called the phantom phase.

iii) $y=y_{0}$. This point is special. At this point $d V / d y=0$ and equations (77) and (8) yield a cosmological constant type EOS

$$
p=-\rho \text {. }
$$

The equilibrium solutions are configurations of constant density $\rho=V\left(y_{0}\right)$. This set of solutions describes the de Sitter phase. Since the chemical potential $\mu$ is now equal to 0 , the condensation is due to the spontaneous symmetry braking rather than due to an excess of positive or negative charge as in the case of Bose-Einstein condensation at $\mu \neq 0$.

\section{Thermodynamics of purely kinetic k-essence}

We will base our thermodynamic analysis on a purely kinetic k-essence action

$$
S=\int d^{4} x \sqrt{-g}\left[-\frac{R}{16 \pi G}+\mathcal{L}(X)\right]
$$

with $\mathcal{L}$ in the form of (18). The Lagrangian $\mathcal{L}$ depends only on variable $X$ defined in (5) with the field $\theta$ of dimension $m^{-1}$. Such theories have been exploited as models for inflation and dark matter/energy, e.g., purely kinetic k-essence [26, 11, 27] or ghost condensate [14, 28, 29]. A perfect fluid description applies for $X>0$. Furthermore, equation (16) implies $\eta W_{X}>$ 0 , which means that the domains where $W_{X}>0$ correspond to a canonical scalar field Lagrangian (11) and those where $W_{X}<0$ to a phantom. In particular, if in the neighborhood of $X=0, W \sim \eta X$, then for $\eta=1$ the kinetic term is canonical and for $\eta=-1$ is of phantom type. The field described by the Lagrangian (18) that behaves as a phantom near $X=0$ is sometimes called a "ghost".

The hydrodynamic quantities associated with eq. (40) are

$$
p=\mathcal{L} ; \quad \rho=2 X \mathcal{L}_{X}-\mathcal{L} .
$$


and the fluid 4-velocity is given by (10, Obviously, the EOS defined by (41) in parametric form is barotropic. Next, we start from the standard thermodynamical relation

$$
d(\rho V)=T d S-p d V
$$

where $V$ is the volume and $S$ is the entropy. If there exist a conserved charge $Q$ with the corresponding charge density $n$ the volume $V=1 / n$, up to a constant factor. Equation (42) may then be written in the form

$$
d \rho=T d s+\mu d n
$$

where $s=S / V$ is the entropy density and we have introduced the chemical potential

$$
\mu=\frac{\rho+p-T s}{n}
$$

associated to the conserved charge $Q$. In this way we define a grand-canonical ensemble in which the thermodynamical quantities $p, \rho$ and $s$ are functions of two variables $\mu$ and $T$. Equation (44) is nothing but the standard grand-canonical expression for the entropy density

$$
s T=p+\rho-\mu n .
$$

Taking the derivative of this equation and combining it with (43) we find that the entropy and charge densities may be expressed as partial derivatives of $p$

$$
s=\left.\frac{\partial p}{\partial T}\right|_{\mu} \quad n=\left.\frac{\partial p}{\partial \mu}\right|_{T}
$$

Using this and (45) we find another useful relation

$$
p+\rho=T \frac{\partial p}{\partial T}+\mu \frac{\partial p}{\partial \mu}
$$

which may help us to narrow the arbitrariness in functional dependence on $T$ and $\mu$. It is clear from (45) that if $\mu=0$ the positivity of entropy requires $p+\rho \geq 0$. Hence, one could conclude that a phantom field must necessarily yield a fluid with a negative entropy. However, this conclusion is incorrect since generally $\mu \neq 0$ and the entropy density given by (45) need not be negative. In fact, as we will shortly demonstrate, for an arbitrary temperature $T$ it is always possible to find a range of $\mu$ such that $s \geq 0$.

From (41) and (47) it follows that the variable $X$ as a function of $T$ and $\mu$ satisfies a partial differential equation

$$
T \frac{\partial X}{\partial T}+\mu \frac{\partial X}{\partial \mu}=2 X .
$$

The most general solution to this equation is a homogeneous function of 2 nd degree which may be written as

$$
X=\frac{\mu^{2}}{m^{2}} f(T / \mu)
$$

or equivalently

$$
X=\frac{T^{2}}{m^{2}} g(\mu / T) .
$$


Here $f$ is an arbitrary positive dimensionless function of $x \equiv T / \mu$ and the function $g$ is related to $f$ by $f(x)=x^{2} g(1 / x)$. The entropy density may be calculated from (46). With (41) and (50) we find

$$
s=X \mathcal{L}_{X} \frac{1}{\mu} \frac{f^{\prime}}{f}
$$

Now we require $S>0$ at $T \neq 0$ and $S=0$ at $T=0$. From the latter requirement it follows $f^{\prime}(0)=0$. The requirement $S \geq 0$ and the condition $\eta \mathcal{L}_{X}>0$ imply $\eta \mu f^{\prime}>0$ for $T \neq 0$. A simple nontrivial function that satisfies the above conditions is, e.g.,

$$
f=C_{1}+\eta C_{2} x^{2}
$$

where $C_{1}$ and $C_{2}$ are arbitrary positive constants. However, in the phantom case the positivity of $f$ puts an additional constraint on $\mu$ and $T$ :

$$
\frac{T}{\mu} \leq \frac{C_{1}}{C_{2}}
$$

\section{Chemical potential}

The equation of motion (19) for the field $\theta$ is in fact a conservation equation for the current

$$
j^{\mu}=2 m^{3} W_{X} g^{\mu \nu} \theta_{, \nu} .
$$

The current conservation is related to the symmetry under the constant shift $\theta \rightarrow \theta+c$ of the scalar field $\theta$. The conserved charge is defined as

$$
Q=\int_{\Sigma} j^{\mu} d \Sigma_{\mu}=\int_{\Sigma} n u^{\mu} d \Sigma_{\mu}
$$

where the integration goes over an arbitrary spacelike hypersurface $\Sigma$ that contains the charge. Using the definition (10) for the velocity, we obtain the charge density as

$$
n=2 m^{3} \sqrt{X} W_{X}
$$

In the Hamiltonian formulation [30] we choose the hypersurface $\Sigma$ at constant time so that the total charge (55) becomes a volume integral

$$
Q=2 m^{3} \int_{V} W_{X} g^{0 \nu} \theta_{, \nu} d V
$$

The current (54) corresponds to the Klein-Gordon current

$$
j_{\mathrm{KG}}^{\mu}=i g^{\mu \nu}\left(\Phi^{*} \Phi_{, \nu}-\Phi \Phi_{, \nu}^{*}\right),
$$

which is the conserved current in the TF equivalent theory.

Next, we introduce the chemical potential $\mu$ associated to the conserved charge (55). To find the effective Lagrangian that contains the chemical potential we start from the grandcanonical partition function

$$
Z=\operatorname{Tr} e^{-\beta(\hat{H}-\mu \hat{Q})}=\int[d \pi] \int_{\text {periodic }}[d \theta] \exp \int_{0}^{\beta} d \tau \int d V\left(i \pi \frac{\partial \theta}{\partial \tau}-\mathcal{H}+\frac{\mu}{m} \pi\right)
$$


where $\beta=1 / T, \pi$ is the conjugate momentum field and the Hamiltonian density $\mathcal{H}$ is related to $\mathcal{L}$ by the usual Legendre transformation. A formal functional integration of (59) over $\pi$ yields the partition function expressed in terms of the effective Euclidean Lagrangian

$$
Z=\int_{\text {periodic }}[d \theta] \exp -\int_{0}^{\beta} d \tau \int d V \mathcal{L}_{\mathrm{E}}(\theta, \mu)
$$

Unfortunately, the analytic functional integration is generally not possible. Nevertheless, in the in the saddle point approximation we find

$$
-\mathcal{L}_{\mathrm{E}}=\mathcal{L}\left(i \frac{\partial \theta}{\partial \tau}+\frac{\mu}{m}, \theta_{, i}\right)
$$

Hence, the effective Lagrangian is obtained from the Lagrangian (18) by replacing the derivatives of the field $\theta$ by

$$
\theta_{, \nu} \rightarrow \theta_{, \nu}+\frac{\mu}{m} \delta_{\nu}^{0}
$$

Note the difference and similarity with the Euclidean field theory prescription (36) for a canonical complex scalar field.

We now check the consistency of the prescription (62) with the solution (49). To do that, it is useful to work in the comoving reference frame i.e., in the frame where the 4-velocity takes the form $u^{\mu}=\delta_{0}^{\mu} / \sqrt{g_{00}}$. Comparing this with the definition of the 4-velocity (10) we conclude that $\theta$ is a function of $t$ only. Then

$$
X=g^{00}\left(\theta_{, 0}+\mu / m\right)^{2} .
$$

This compared with the general expression (49) implies $\theta_{,_{0}}=0, T=0$, and $S=0$. Hence, we conclude that the consistency of (62) with (49) implies zero temperature and zero entropy for a general purely kinetic k-essence type of theory.

Two remarks are in order. First, one should bear in mind that this result is obtained using the effective Euclidean Lagrangian (61) derived in a saddle point approximation. Second, the solution (49) is classical whereas the partition function (60) generally represents quantum and thermal fluctuations of the field $\theta$.

\section{Summary and conclusion}

Using the Thomas Fermi correspondence we have shown that a general DE model based on a complex scalar field theory can be equivalently represented by a purely kinetic k-essence modell. Our thermodynamic analysis of purely kinetic k-essence shows that the entropy is positive or zero and need not be negative for phantom theories contrary to the claims often stated in the recent literature (see, e.g., [8] and references therein) that a violation of NEC implies negative entropy. Furthermore, using the grandcanonical partition function derived in a saddle point approximation of the functional integral over conjugate momenta and comparing the effective Euclidean Lagrangian with the quintessence equation of state we obtain quite generally the chemical potential $\mu \neq 0$ but the temperature $T=0$ and the entropy $S=0$. In principle, nontrivial thermal contributions can be obtained from quadratic fluctuations of the field around the classical solution. However this would go beyond the scope of this paper and will be done elswhere. 


\section{Acknowledgments}

This work was supported by the Ministry of Science, Education and Sport of the Republic of Croatia under contract No. 098-0982930-2864 and partially supported through the Agreement between the Astrophysical Sector, S.I.S.S.A., and the Particle Physics and Cosmology Group, RBI.

\section{References}

[1] I. Brevik, S. Nojiri, S. D. Odintsov and L. Vanzo, Phys. Rev. D 70 (2004) 043520 arXiv:hep-th/0401073; S. Nojiri and S. D. Odintsov, Phys. Rev. D 70 (2004) 103522 arXiv:hep-th/0408170.

[2] J. A. S. Lima and J. S. Alcaniz, Phys. Lett. B 600 (2004) 191 arXiv:astro-ph/0402265.

[3] P. F. Gonzalez-Diaz and C. L. Siguenza, Nucl. Phys. B 697 (2004) 363 arXiv:astro-ph/0407421.

[4] G. Izquierdo and D. Pavon, Phys. Lett. B 633 (2006) 420 arXiv:astro-ph/0505601.

[5] H. Mohseni Sadjadi, Phys. Rev. D 73 (2006) 063525 arXiv:gr-qc/0512140.

[6] M. R. Setare and S. Shafei, JCAP 0609 (2006) 011 [arXiv:gr-qc/0606103]; M. R. Setare, Phys. Lett. B 641 (2006) 130 [arXiv:hep-th/0611165].

[7] B. Wang, Y. Gong and E. Abdalla, Phys. Rev. D 74 (2006) 083520 arXiv:gr-qc/0511051.

[8] Y. Gong, B. Wang and A. Wang, Phys. Rev. D 75 (2007) 123516 arXiv:gr-qc/0611155.

[9] F. C. Santos, M. L. Bedran and V. Soares, Phys. Lett. B 636 (2006) 86; Phys. Lett. B 646 (2007) 215.

[10] C. Wetterich, Nucl. Phys. B 302, 668 (1988); P.J.E. Peebles and B. Ratra, Astrophys. J. 325, L17 (1988).

[11] C. Armendariz-Picon, V. F. Mukhanov and P. J. Steinhardt, Phys. Rev. Lett. 85 (2000) 4438

[12] E. J. Copeland, M. Sami and S. Tsujikawa, Int. J. Mod. Phys. D 15 (2006) 1753 arXiv:hep-th/0603057.

[13] The terminology is borrowed from Bose-Einstein condensates: A.S. Parkins and D.F. Walls, Phys. Rep. 303, 1 (1998).

[14] N. Arkani-Hamed, H.C. Cheng, M.A. Luty, and S. Mukohyama, JHEP 05 (2004) 074.

[15] N. Bilić, G.B. Tupper, and R.D. Viollier, Phys. Lett. B535 (2002) 17.

[16] A. Kamenshchik, U. Moschella, and V. Pasquier, Phys. Lett. B511 (2001) 265. 
[17] J. C. Fabris, S. V. B. Goncalves and P. E. De Souza, Gen. Rel. Grav. 34 (2002) 53; Gen. Rel. Grav. 34 (2002) 2111.

[18] M.C. Bento, O. Bertolami, and A.A. Sen, Phys. Rev. D66 (2002) 043507.

[19] M. Makler, S.Q. de Oliveira, and I. Waga, Phys. Lett. B555 (2003) 1.

[20] M. Bordemann and J. Hoppe, Phys. Lett. B325 (1994) 359; N. Ogawa, Phys. Rev. D62 (2000) 085023.

[21] R. Jackiw, Lectures on Fluid Mechanics (Springer Verlag, Berlin, 2002).

[22] N. Bilić, G.B. Tupper, and R.D. Viollier, J. Phys. A 40 (2007) 6877; gr-qc/0610104.

[23] C.V. Johnson, D-Branes, Cambridge University Press, Cambridge, 2003.

[24] M. Laine and M. Shaposhnikov, Nucl. Phys. B 532 (1998) 376.

[25] N. Bilić and H. Nikolić, Nucl Phys. B 590 (2000) 575

[26] C. Armendariz-Picon, T. Damour and V. F. Mukhanov, Phys. Lett. B 458 (1999) 209 arXiv:hep-th/9904075.

[27] R.J. Scherrer, Phys. Rev. Lett. 93 (2004) 011301.

[28] D. Krotov, C. Rebbi, V. A. Rubakov and V. Zakharov, Phys. Rev. D 71 (2005) 045014, arXiv:hep-ph/0407081.

[29] A. Krause and S. P. Ng, Int. J. Mod. Phys. A 21 (2006) 1091, arXiv:hep-th/0409241.

[30] R.M. Wald, General relativity, University of Chicago, Chicago 1984. 